\title{
BLACK HOLE MASSES OF HIGH-REDSHIFT QUASARS
}

\author{
M. VESTERGAARD \\ Steward Observatory \\ 933 N. Cherry Avenue, \\ Tucson, AZ, 85721, USA \\ E-mail: mvestergaard@as.arizona.edu
}

\begin{abstract}
Black-hole masses of distant quasars cannot be measured directly, but can be estimated to within a factor 3 to 5 using scaling relationships involving the quasar luminosity and broad-line width. Why such relationships are reasonable is summarized. The results of applying scaling relationships to data of quasars at a range of redshifts $(z \leq 6.3)$ are presented. Luminous quasars typically have masses $\sim 10^{9} M_{\odot}$ even at the highest redshifts. The fact that such massive black holes appear as early as at $z \approx 6$ indicate that black holes form very early or build up mass very fast.
\end{abstract}

\section{Introduction}

With our recently acquired ability to measure black-hole masses in nearby quiescent and active galaxies we are now in a position to start addressing the important issues of the physics of black-hole evolution and the possible role of black holes in how galaxies evolve. An important first step is to establish the typical mass of black holes in AGNs at high redshift relative to more nearby AGNs. Such a study ${ }^{1}$ is summarized here.

\section{Mass Estimates}

The most robust method to determine the mass of the central black hole in active galaxies is that of reverberation mapping. However, this method is impractical for large samples of luminous and distant quasars as it takes many years to measure quasar masses. The reasons are that luminous quasars vary with smaller amplitudes and on longer time scales that are further increased by time dilation due to their cosmological distances. Mass estimates based on single-epoch data are therefore very useful, even if less accurate. Of the "secondary mass estimators" used in the literature only a couple are useful at high redshift, as reviewed by Vestergaard" ${ }^{2}$. The "scaling 
relations" used here also appear the most promising at present given their relatively lower and readily quantifiable associated uncertainties ${ }^{2}$.

Scaling relations are approximations to the virial mass $\left(M \propto v^{2} R\right)$ determination of the reverberation mapping method, where the light travel time delay, $\tau=R / c$, between continuum and line variations determine the distance $R$ of the line-emitting gas and the line width of the variable part of the line profile, the RMS profile, yields the velocity dispersion $v$ of the same varying gas. Instead, scaling relations use the empirical radius - luminosity relationship ${ }^{3}$, where $R \propto L^{0.7}$, and single-epoch measurements of the line width and the continuum luminosity to estimate the black-hole mass. Vestergaard ${ }^{4}$ calibrated single-epoch mass estimates based on $\mathrm{H} \beta$ and $\mathrm{C}$ IV line widths, respectively, to the more accurate reverberation masses. The associated statistical uncertainty is a factor $\sim 3$ relative to the reverberation masses (i.e., a factor $\sim 5$ on an absolute scale). However, mass estimates of individual objects can be in error up to a factor of 10 . The mass estimates presented below are based on these two relations.

Contrary to the belief of some, scaling relations are reasonable, even those based on UV spectroscopy for the following reasons. First, contemporaneous UV-optical monitoring of NGC 5548, the best-studied nearby Seyfert, shows that all the broad lines measured (Si IV $\lambda 1400$, CiV $\lambda 1549$, He II $\lambda 1640$, C III] $\lambda 1909, \mathrm{H} \beta \lambda 4861$, He II $\lambda 4686)$ are consistent with virial motion of the broad-line region ${ }^{5,6}$ : higher ionization lines are emitted closer to the central source and have larger Doppler widths. Second, this virial relationship is seen for all (four) AGNs that can be tested ${ }^{5,6,7}$ and so it is fair to assume the relationship is universal, even if the sample is small. Third, since the virial product $\left(v^{2} \tau\right)$ is constant for each AGN, the velocity dispersions and the response-lag scale between the emission lines. Finally, the $R-L$ relation extends also to high redshift and high luminosity quasars because (1) quasar spectra are very similar ${ }^{8}$ at all redshifts and luminosities considered here, and (2) the most luminous quasars have luminosities not much larger $(\lesssim 1.5 \mathrm{dex})$ than the luminosity range over which the $R-L$ relationship is defined ${ }^{1}$. See Vestergaard ${ }^{1,2,9}$ for further details.

\section{Quasar Samples and Data}

In the following, mass estimates are presented for different samples of quasars spanning the redshift range $0 \lesssim z \lesssim 6.2$ : the Bright Quasar Survey (BQS) of 87 objects at $z \leq 0.5$, a sample of $1141.5 \lesssim z \lesssim 3.5$ quasars almost equally distributed among radio-quiet and radio-loud sources, and 

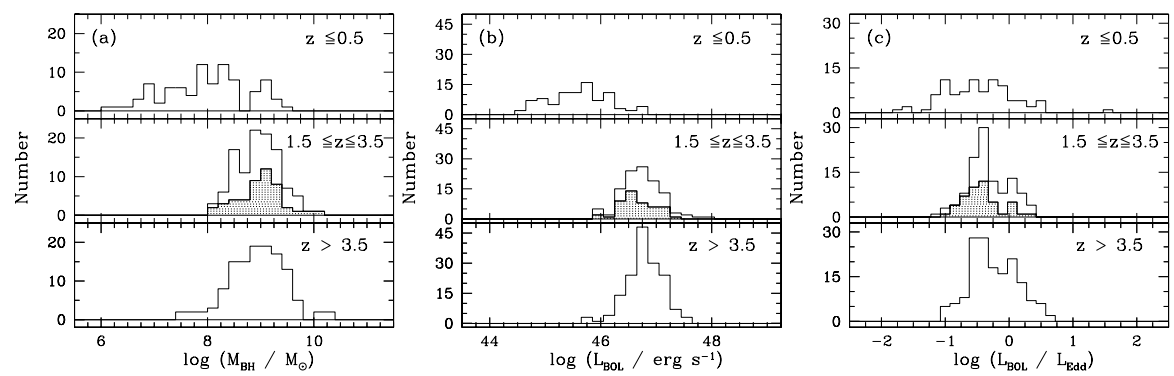

Figure 1. Distributions of estimated (a) black-hole mass, (b) bolometric luminosity, and (c) Eddington ratio for different redshift bins $\left(H_{0}=75 \mathrm{~km} \mathrm{~s}^{-1} \mathrm{Mpc}^{-1}, \mathrm{q}_{0}=0.5\right.$, and $\Lambda=0)$. In the middle panel the radio-quiet subset is shown shaded to illustrate that the two radio types do not differ in these parameters as claimed earlier (e.g., [12]).

$\sim 150 z \approx 4$ quasars from recently published samples which include objects and data from the Sloan Digital Sky Survey. See Vestergaard ${ }^{1}$ for details. Bolometric luminosities were estimated using bolometric corrections to restframe UV luminosities, based on average spectral energy distributions ${ }^{10}$, updated to include a more realistic X-ray energy distribution ${ }^{11}$.

\section{Masses of Distant Quasars}

Figure 1 shows that the luminous quasars at $z>1.5$ are similarly distributed in black-hole mass $M_{\mathrm{BH}}$, bolometric luminosity $L_{\mathrm{bol}}$, and Eddington ratios $\left(L_{\mathrm{bol}} / L_{\mathrm{Edd}}\right)$ with an average mass of $10^{9} M_{\odot}$ and luminosity of $10^{47} \mathrm{ergs} \mathrm{s}^{-1}$. While the lower limits in $M_{\mathrm{BH}}$ and $L_{\mathrm{bol}}$ are due to the sample selection and survey limits, the data show the important fact that the luminous, distant quasars that we can detect are equally massive as the lower redshift quasars, even beyond the epoch $(z \gtrsim 3)$ where the comoving quasar space density drops. Moreover, there are characteristic, but real, ceilings at $M_{\mathrm{BH}} \approx 10^{10} M_{\odot}$ and $L_{\mathrm{bol}} \approx 10^{48} \mathrm{ergs} \mathrm{s}^{-1}$.

\section{Mass Functions}

Vestergaard, Osmer, and Fan (in preparation) are currently determining mass functions of active black holes at different redshifts. Our first results show that the BQS, Large Bright Quasar Survey (LBQS), and colorselected SDSS samples exhibit consistent mass functions (Fig. 2; $H_{0}=50$ $\left.\mathrm{km} \mathrm{s}^{-1} \mathrm{Mpc}^{-1}, \mathrm{q}_{0}=0.5, \Lambda=0\right)$. The goal is to constrain black-hole growth by combining theoretical models (see Steed this volume) with measurements from large data bases. 
388

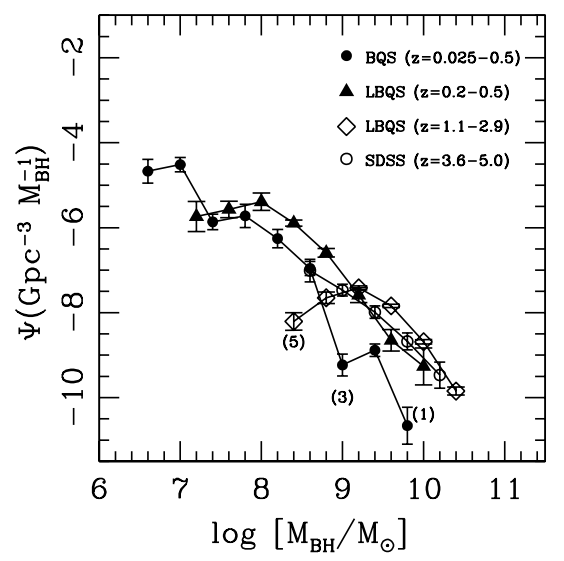

Figure 2. Mass functions of the BQS, LBQS (preliminary), and the color-selected SDSS sample $\left(H_{0}=50 \mathrm{~km} \mathrm{~s}^{-1} \mathrm{Mpc}^{-1}, \mathrm{q}_{0}=0.5, \Lambda=0\right)$. The low-mass turn-down for more distant LBQS quasars is an artifact owing to the lower flux-limit of the sample.

\section{Conclusions}

The two main conclusions from this work are:

(1) Black-hole masses in nearby AGNs can be measured to within a factor 3. For more distant AGNs, useful mass estimates can be obtained to within a factor of 3 to 5 . Even if less accurate, mass estimates are particularly useful for statistical studies.

(2) Black holes of luminous quasars are very massive $\left(\sim 10^{9} M_{\odot}\right)$ even at the highest redshifts of 4 to 6 . The existence of such massive black holes at these early epochs indicate that they formed very early or very fast.

\section{References}

1. M. Vestergaard, Astroph. Journal 601, 676 (2004)

2. M. Vestergaard, preprint (astro-ph/0401436) (2004)

3. S. Kaspi et al., Astroph. Journal 533, 631 (2000)

4. M. Vestergaard, Astroph. Journal 571, 733 (2002)

5. B.M. Peterson \& A. Wandel, Astroph. Journal 521, L95 (1999)

6. B.M. Peterson \& A. Wandel, Astroph. Journal 540, L13 (2000)

7. C. Onken \& B.M. Peterson, Astroph. Journal 572, 746 (2002)

8. M. Dietrich et al., Astroph. Journal 581, 912 (2002)

9. M. Vestergaard, preprint (astro-ph/0401430) (2004)

10. M. Elvis, et al., Astroph. Journal Suppl. 95, 1 (1994)

11. M. Elvis, G. Risaliti, \& G. Zamorani, Astroph. Journal 565, L75 (2002)

12. A. Laor, Astroph. Journal 543, L111 (2000) 